\title{
Synthesis and Fluorescence Spectra of Oxa[3.n]phenanthrenophanes
}

\author{
Yosuke Nakamura, Takuzo Yamazaki, and Jun Nishimura* \\ Department of Nano-Material Systems, Graduate School of Engineering, Gunma University, \\ Tenjin-cho, Kiryu, Gunma, 376-8515, Japan
}

\section{Supporting Information}

Spectroscopic data of anti-6a, anti-6b, syn-6b, anti-7, and syn-7 are as follows.

anti-6a: mp 203-205 ${ }^{\circ} \mathrm{C} . \quad{ }^{1} \mathrm{H}$ NMR $\left(500 \mathrm{MHz}, \mathrm{CDCl}_{3}\right) \delta 8.44(2 \mathrm{H}, \mathrm{d}, J=8.3 \mathrm{~Hz}, \mathrm{H} 5), 8.19$ (2H, d, $J=8.0 \mathrm{~Hz}, \mathrm{H} 8), 7.80$ (2H, s, H4), 7.69 (4H, m, H6 and H7), 7.19 (2H, d, $J=8.3 \mathrm{~Hz}$, H2), 6.52 (2H, d, $J=8.0 \mathrm{~Hz}, \mathrm{H} 1), 6.19$ (2H, s, H10), 4.88 (4H, bs, $\left.\mathrm{ArCH}_{2} \mathrm{O}\right), 3.56(2 \mathrm{H}, \mathrm{m}$, $\left.\mathrm{ArCH}_{2} \mathrm{CH}_{2}\right), 2.82\left(2 \mathrm{H}, \mathrm{m}, \mathrm{ArCH}_{2} \mathrm{CH}_{2}\right), 2.52\left(2 \mathrm{H}, \mathrm{m}, \mathrm{ArCH}_{2} \mathrm{CH}_{2}\right) .{ }^{13} \mathrm{C} \mathrm{NMR}(125 \mathrm{MHz}$, $\left.\mathrm{CDCl}_{3}\right) \delta 135.34$ (C), 135.12 (C), 131.18 (C), 130.52 (C), 130.16 (C), 129.06 (C), 126.31 (CH; overlap of two signals), $126.09(\mathrm{CH}), 125.47(\mathrm{CH}), 124.98(\mathrm{CH}), 124.13(\mathrm{CH}), 123.47$ $(\mathrm{CH}), 123.33(\mathrm{CH}), 76.48\left(\mathrm{CH}_{2}\right), 32.27\left(\mathrm{CH}_{2}\right), 24.46\left(\mathrm{CH}_{2}\right)$. HRMS (EI) calcd for $\mathrm{C}_{33} \mathrm{H}_{26} \mathrm{O}$ $\left(\mathrm{M}^{+}\right)$438.1984; found 438.1985.

anti-6b: mp 219-220 ${ }^{\circ} \mathrm{C} . \quad{ }^{1} \mathrm{H}$ NMR $\left(500 \mathrm{MHz}, \mathrm{CDCl}_{3}\right) \delta 8.42(2 \mathrm{H}, \mathrm{d}, J=9.2 \mathrm{~Hz}, \mathrm{H} 5), 7.94$ (2H, d, $J=9.2 \mathrm{~Hz}, \mathrm{H} 8), 7.84$ (2H, s, H4), 7.63 (4H, m, H6 and H7), 7.27 (2H, d, $J=8.2 \mathrm{~Hz}$, H2), 6.65 (2H, d, J = 7.9 Hz, H1), 6.21 (2H, s, H10), $4.95\left(4 \mathrm{H}, \mathrm{s}, \mathrm{ArCH}_{2} \mathrm{O}\right), 3.31(2 \mathrm{H}, \mathrm{m}$, $\left.\mathrm{ArCH}_{2} \mathrm{CH}_{2}\right), 2.17\left(4 \mathrm{H}, \mathrm{m}, \mathrm{ArCH}_{2} \mathrm{CH}_{2}\right), 1.67\left(2 \mathrm{H}, \mathrm{m}, \mathrm{ArCH}_{2} \mathrm{CH}_{2}\right) .{ }^{13} \mathrm{C} \mathrm{NMR}(125 \mathrm{MHz}$, $\left.\mathrm{CDCl}_{3}\right) \delta 135.81$ (C), 135.70 (C), 130.94 (C), 130.34 (C), 130.22 (C), 128.41 (C), 126.41 $(\mathrm{CH}), 126.26(\mathrm{CH}), 125.93(\mathrm{CH}), 125.35(\mathrm{CH}), 125.24(\mathrm{CH}), 124.42(\mathrm{CH}), 123.28(\mathrm{CH})$, $122.83(\mathrm{CH}), 76.37\left(\mathrm{CH}_{2}\right), 33.25\left(\mathrm{CH}_{2}\right), 29.96\left(\mathrm{CH}_{2}\right)$. HRMS (EI) calcd for $\mathrm{C}_{34} \mathrm{H}_{28} \mathrm{O}\left(\mathrm{M}^{+}\right)$ 452.2140; found 452.2115 .

syn-6b: mp 232-233 ${ }^{\circ} \mathrm{C} . \quad{ }^{1} \mathrm{H}$ NMR (500 MHz, $\left.\mathrm{CDCl}_{3}\right) \delta 8.21(2 \mathrm{H}, \mathrm{d}, J=8.0 \mathrm{~Hz}, \mathrm{H} 5), 8.09$ (2H, s, H4), 7.55 (2H, d, $J=7.7$ Hz, H8), 7.19-7.10 (4H, m, H6 and H7), 7.17 (4H, s, H1 and H2), 6.98 (2H, s, H10), 4.99 (4H, ABq, $\left.\mathrm{ArCH}_{2} \mathrm{O}\right), 3.15$ (2H, m, $\left.\mathrm{ArCH}_{2} \mathrm{CH}_{2}\right), 2.43(2 \mathrm{H}, \mathrm{m}$, $\left.\mathrm{ArCH}_{2} \mathrm{CH}_{2}\right), 1.94\left(2 \mathrm{H}, \mathrm{m}, \mathrm{ArCH}_{2} \mathrm{CH}_{2}\right), 1.79\left(2 \mathrm{H}, \mathrm{m}, \mathrm{ArCH}_{2} \mathrm{CH}_{2}\right) .{ }^{13} \mathrm{C}$ NMR $(125 \mathrm{MHz}$, $\left.\mathrm{CDCl}_{3}\right) \delta$ 136.36(C), 135.71(C), 135.16(C), 130.24(C), 129.73 (C), 127.38 (CH; overlap of two signals), $125.87(\mathrm{CH}), 125.48(\mathrm{CH}), 125.44(\mathrm{CH}), 123.74(\mathrm{CH}), 123.45(\mathrm{CH}), 122.44$ (CH), $76.24\left(\mathrm{CH}_{2}\right), 30.95\left(\mathrm{CH}_{2}\right), 30.23\left(\mathrm{CH}_{2}\right)$. (One quaternary carbon peak is missing probably due to the overlap.) HRMS (EI) calcd for $\mathrm{C}_{34} \mathrm{H}_{28} \mathrm{O}\left(\mathrm{M}^{+}\right)$452.2140; found 452.2123 .

anti-7: mp 217-218 ${ }^{\circ} \mathrm{C} . \quad{ }^{1} \mathrm{H}$ NMR (500 MHz, $\left.\mathrm{CDCl}_{3}\right) \delta 8.68(2 \mathrm{H}, \mathrm{d}, J=7.7 \mathrm{~Hz}, \mathrm{H} 5), 8.51$ (2H, s, H4), 7.91 (2H, d, $J=8.2 \mathrm{~Hz}, \mathrm{H} 8), 7.74$ (2H, s, H9), 7.64 (4H, m, H6 and H7), 6.36 $(2 \mathrm{H}, \mathrm{d}, J=8.7 \mathrm{~Hz}, \mathrm{H} 1), 6.32(2 \mathrm{H}, \mathrm{d}, J=8.7 \mathrm{~Hz}, \mathrm{H} 2), 4.87\left(4 \mathrm{H}, \mathrm{ABq}, \mathrm{ArCH}_{2} \mathrm{O}\right), 2.83(4 \mathrm{H}, \mathrm{m}$, $\mathrm{ArCH}_{2} \mathrm{CH}_{2}$ ), $2.60\left(2 \mathrm{H}, \mathrm{m}, \mathrm{ArCH}_{2} \mathrm{CH}_{2}\right) .{ }^{13} \mathrm{C} \mathrm{NMR}\left(125 \mathrm{MHz}, \mathrm{CDCl}_{3}\right) \delta 136.69(\mathrm{C}), 135.79$ 
(C), 131.83 (C), 131.08 (C), 129.79 (C), 129.33 (C), 127.94 (CH), 127.37 (CH), 126.40 (CH), $125.79(\mathrm{CH}), 125.38(\mathrm{CH}), 123.41(\mathrm{CH}), 122.66(\mathrm{CH}), 122.50(\mathrm{CH}), 75.72\left(\mathrm{CH}_{2}\right), 31.91$ $\left(\mathrm{CH}_{2}\right), 24.25\left(\mathrm{CH}_{2}\right)$. HRMS (EI) calcd for $\mathrm{C}_{33} \mathrm{H}_{26} \mathrm{O}\left(\mathrm{M}^{+}\right)$438.1984; found 438.1972.

syn-7: mp 247-248 ${ }^{\circ} \mathrm{C} . \quad{ }^{1} \mathrm{H}$ NMR (500 MHz, $\left.\mathrm{CDCl}_{3}\right) \delta 8.02(2 \mathrm{H}, \mathrm{s}, \mathrm{H} 4), 8.01(2 \mathrm{H}, \mathrm{d}, J=9.5$ Hz, H5), 7.70 (2H, d, $J=8.2 \mathrm{~Hz}, \mathrm{H} 1), 7.28$ (2H, d, $J=8.2 \mathrm{~Hz}, \mathrm{H} 2), 7.21$ (2H, d, $J=6.7 \mathrm{~Hz}$, H8), 7.12-7.05 (4H, m, H6 and H7), 6.99 (2H, s, H9), 4.97 (4H, Abq, $\left.\mathrm{ArCH}_{2} \mathrm{O}\right), 3.65$ (4H, m, $\left.\mathrm{ArCH}_{2} \mathrm{CH}_{2}\right), 3.27\left(1 \mathrm{H}, \mathrm{m}, \mathrm{ArCH}_{2} \mathrm{CH}_{2}\right), 2.30\left(1 \mathrm{H}, \mathrm{m}, \mathrm{ArCH}_{2} \mathrm{CH}_{2} .{ }^{13} \mathrm{C} \mathrm{NMR}(125 \mathrm{MHz}\right.$, $\left.\mathrm{CDCl}_{3}\right) \delta 135.43$ (C), 134.45 (C), 131.39 (C), 129.83 (C), 129.46 (C), 128.84 (C), 128.72 $(\mathrm{CH}), 127.25(\mathrm{CH}), 126.95(\mathrm{CH}), 125.88(\mathrm{CH}), 125.25(\mathrm{CH}), 125.15(\mathrm{CH}), 124.16(\mathrm{CH})$, $121.40(\mathrm{CH}), 75.96(\mathrm{CH}), 35.54\left(\mathrm{CH}_{2}\right), 29.71\left(\mathrm{CH}_{2}\right)$. HRMS (EI) calcd for $\mathrm{C}_{33} \mathrm{H}_{26} \mathrm{O}\left(\mathrm{M}^{+}\right)$ 438.1984; found 438.1962. 
${ }^{1} \mathrm{H} \mathrm{NMR}$ of anti-6a in $\mathrm{CDCl}_{3}$

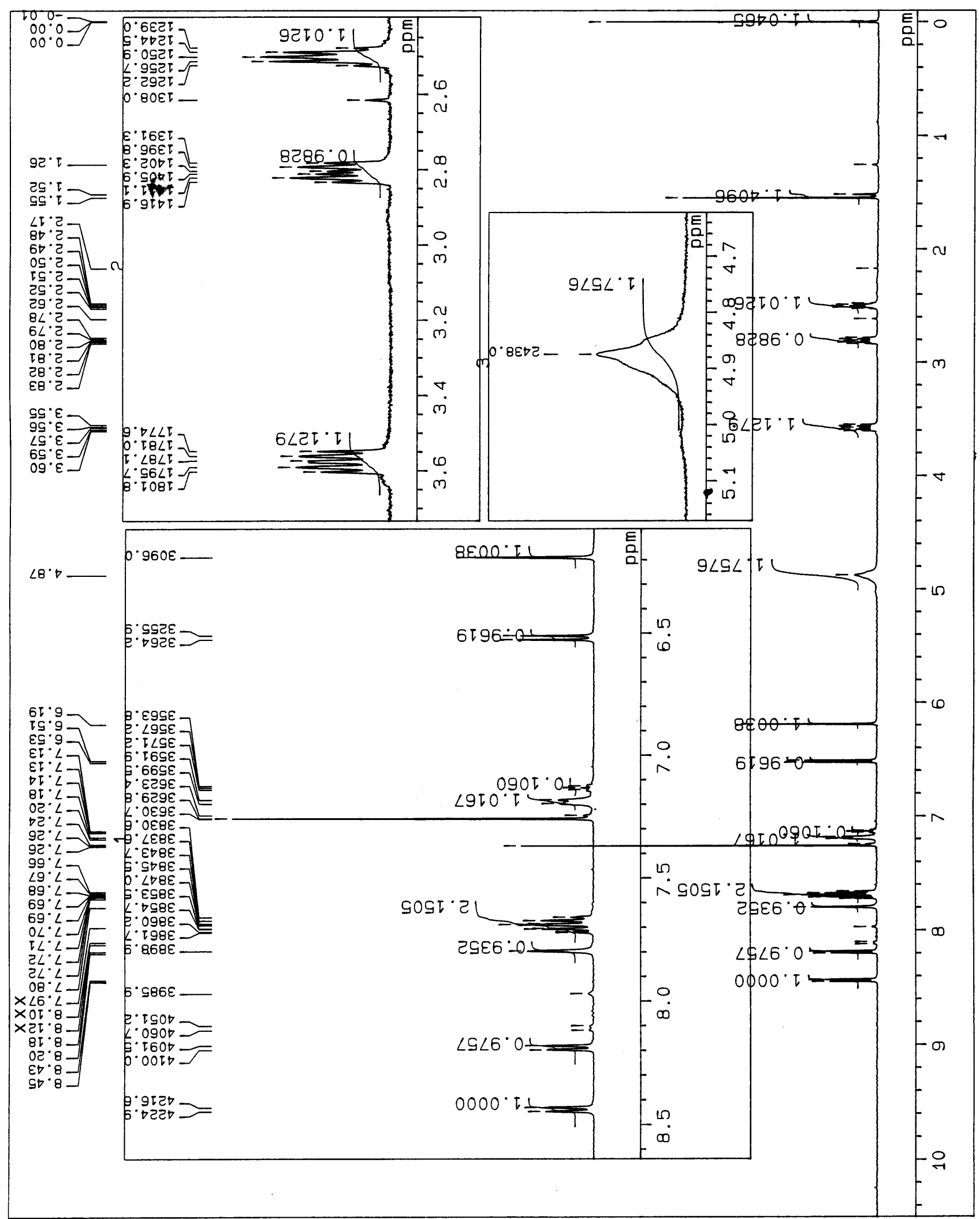


${ }^{13} \mathrm{C}$ NMR of anti-6a in $\mathrm{CDCl}_{3}$

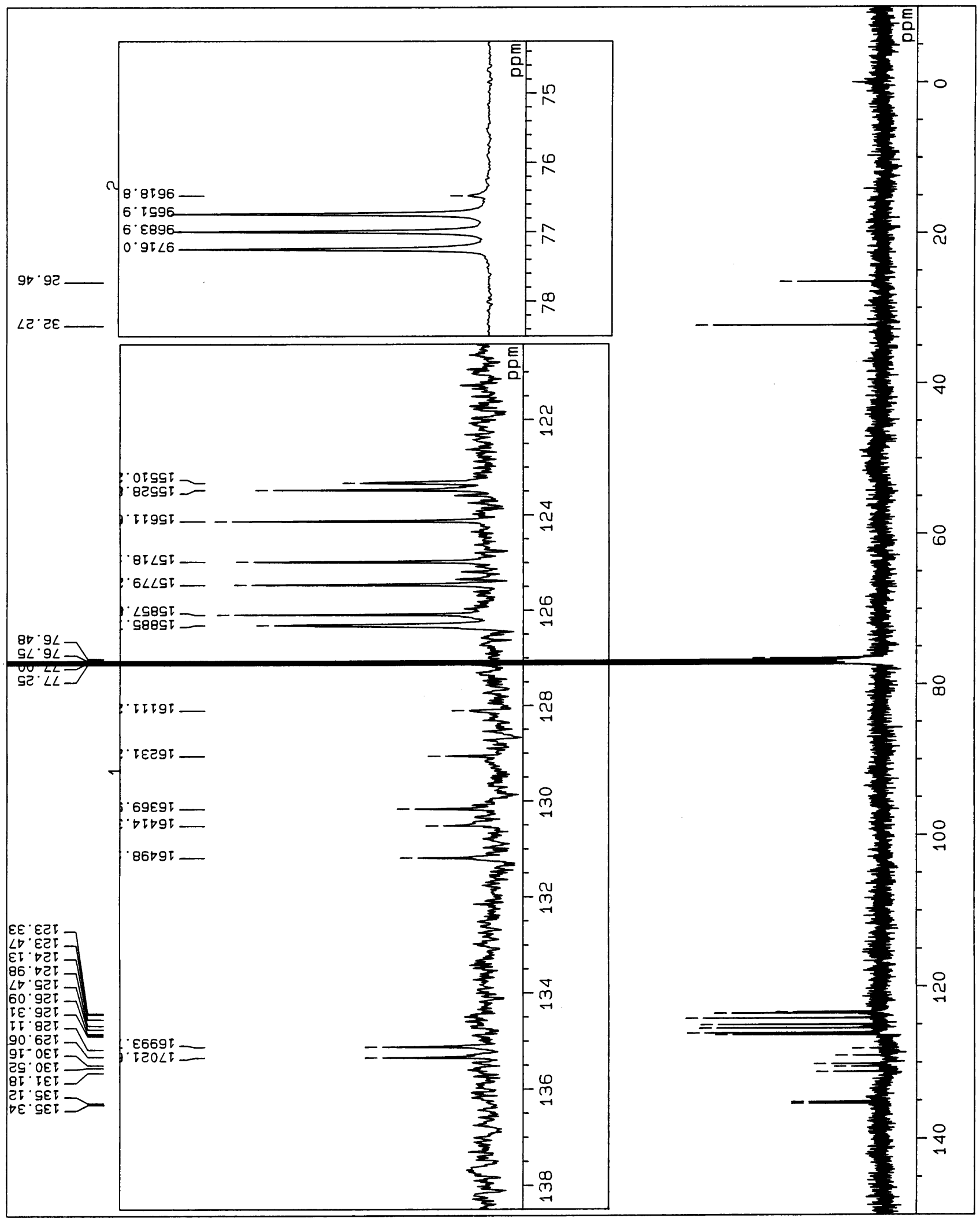


${ }^{1} \mathrm{H}$ NMR of anti-6b in $\mathrm{CDCl}_{3}$

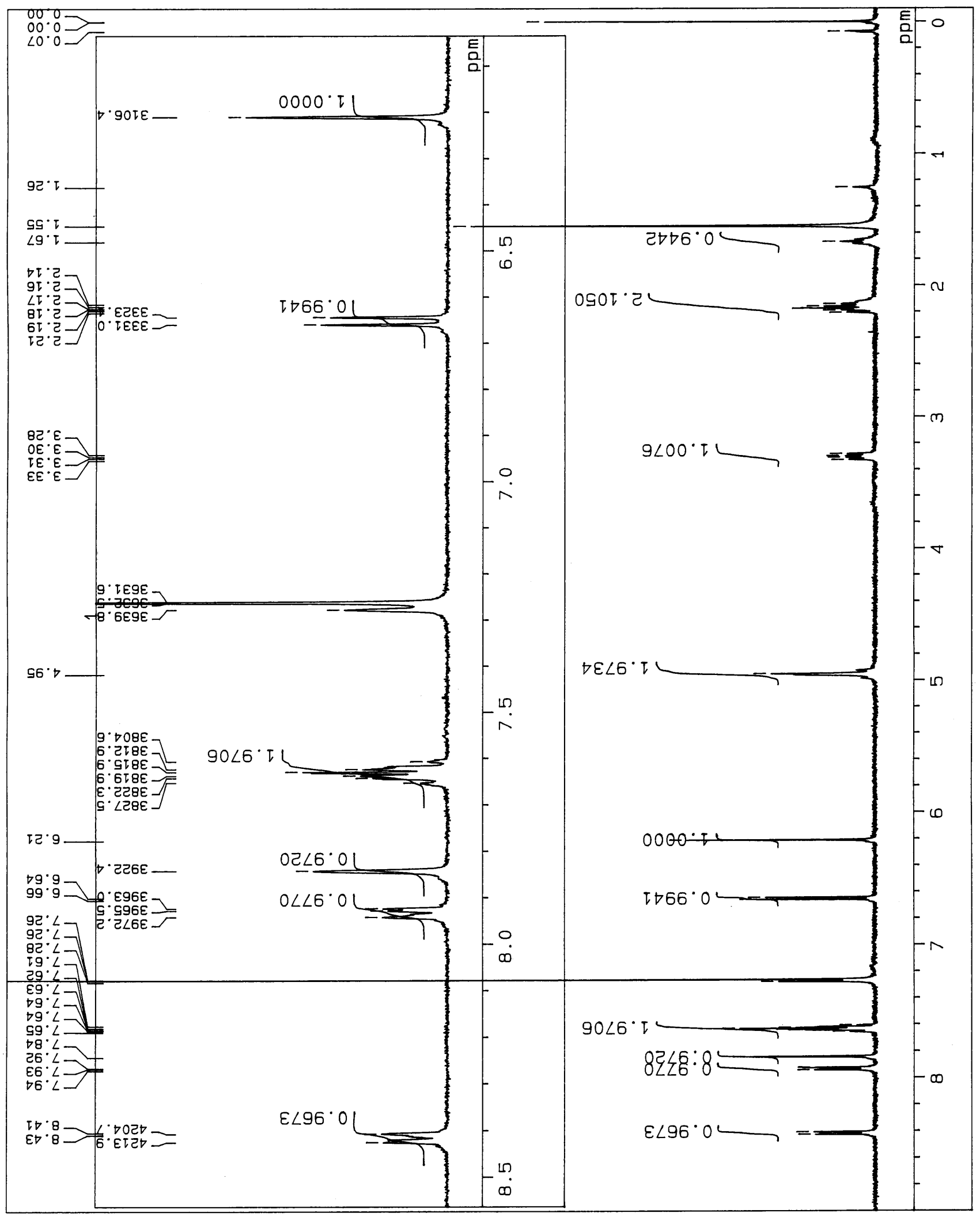


${ }^{13} \mathrm{C}$ NMR of anti-6b in $\mathrm{CDCl}_{3}$

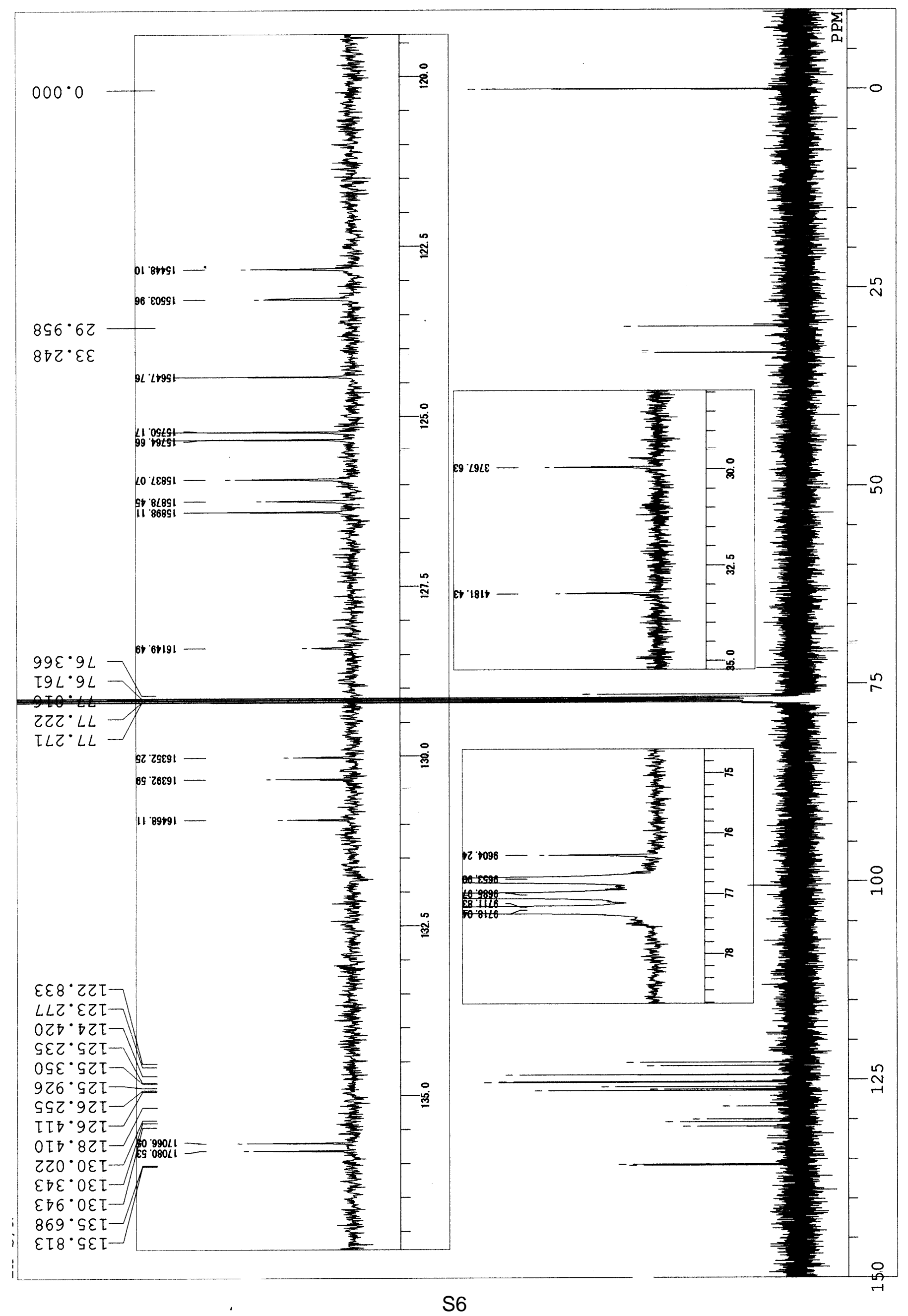


${ }^{1} \mathrm{H}$ NMR of syn-6b in $\mathrm{CDCl}_{3}$

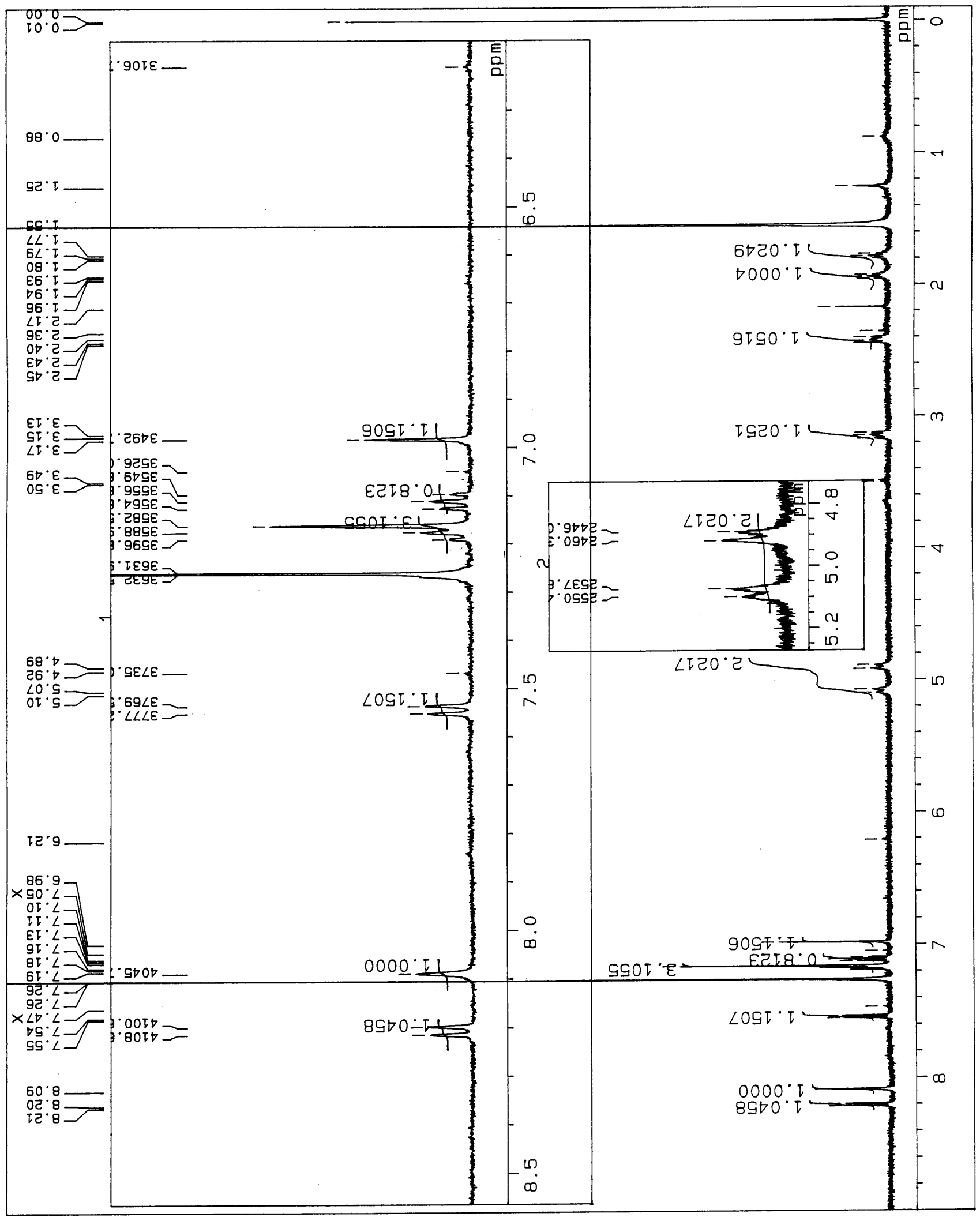


${ }^{13} \mathrm{C} \mathrm{NMR}$ of $s y n-6 \mathbf{b}$ in $\mathrm{CDCl}_{3}$

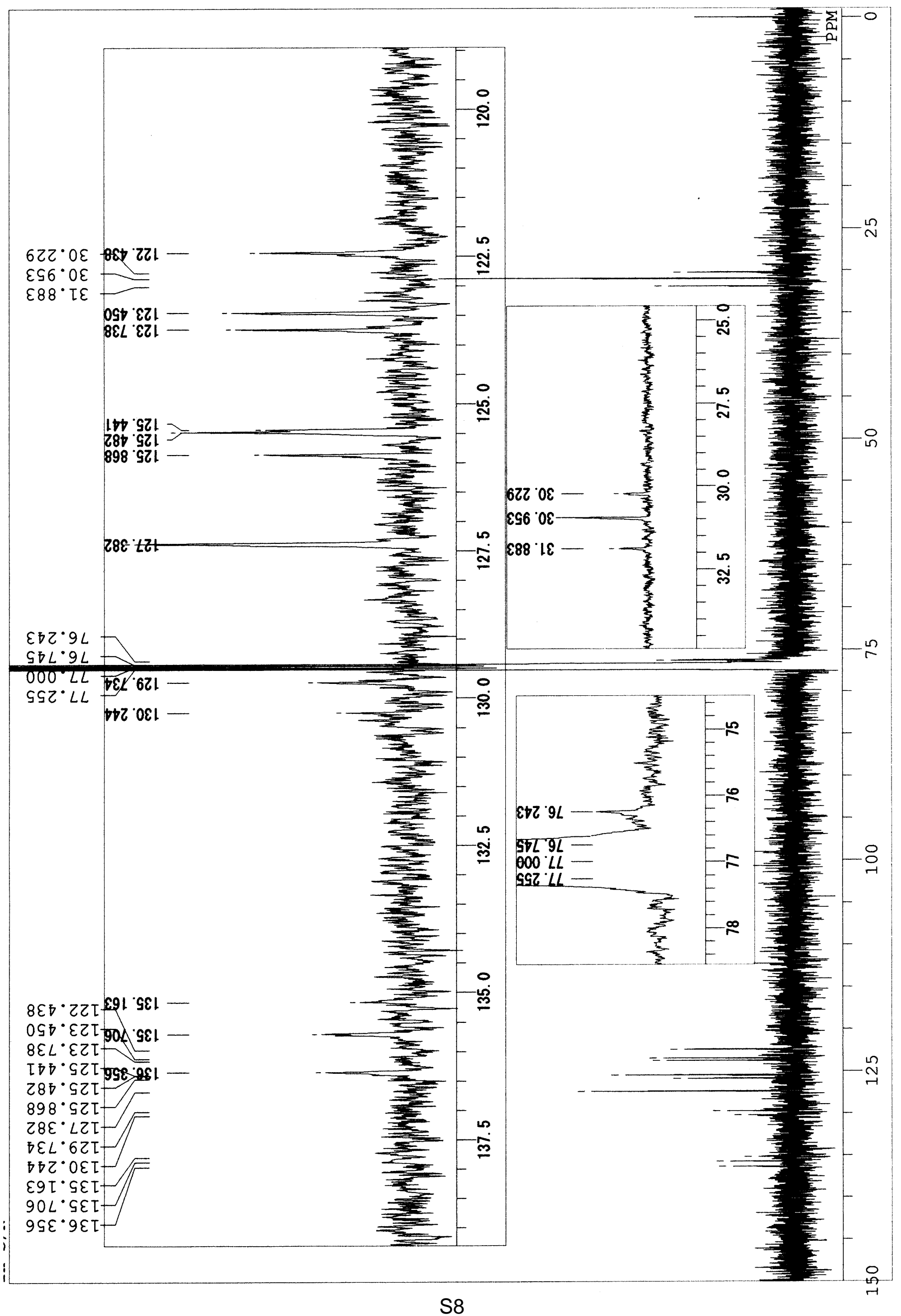


${ }^{1} \mathrm{H}$ NMR of anti-7 in $\mathrm{CDCl}_{3}$

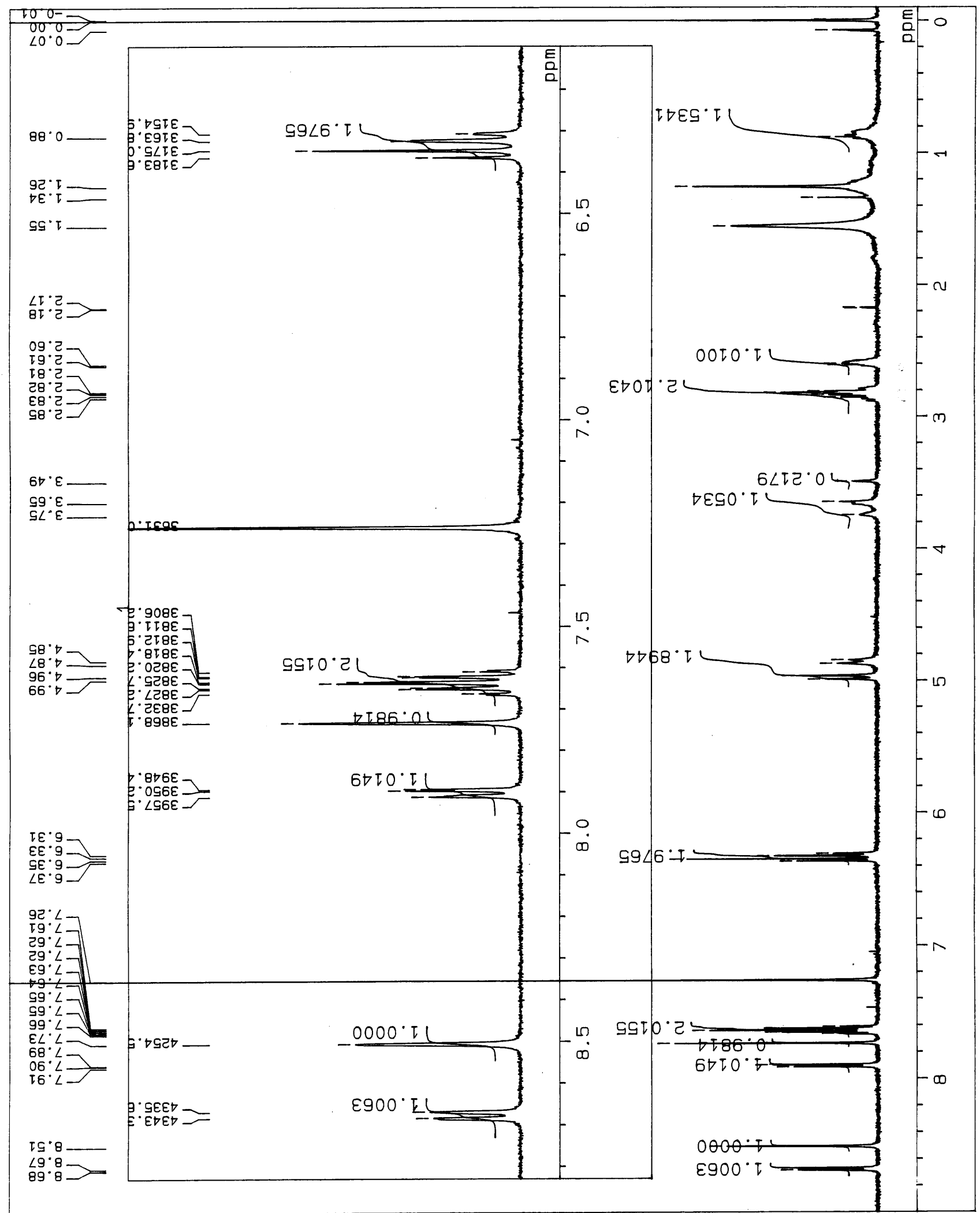


${ }^{13} \mathrm{C}$ NMR of anti-7 in $\mathrm{CDCl}_{3}$

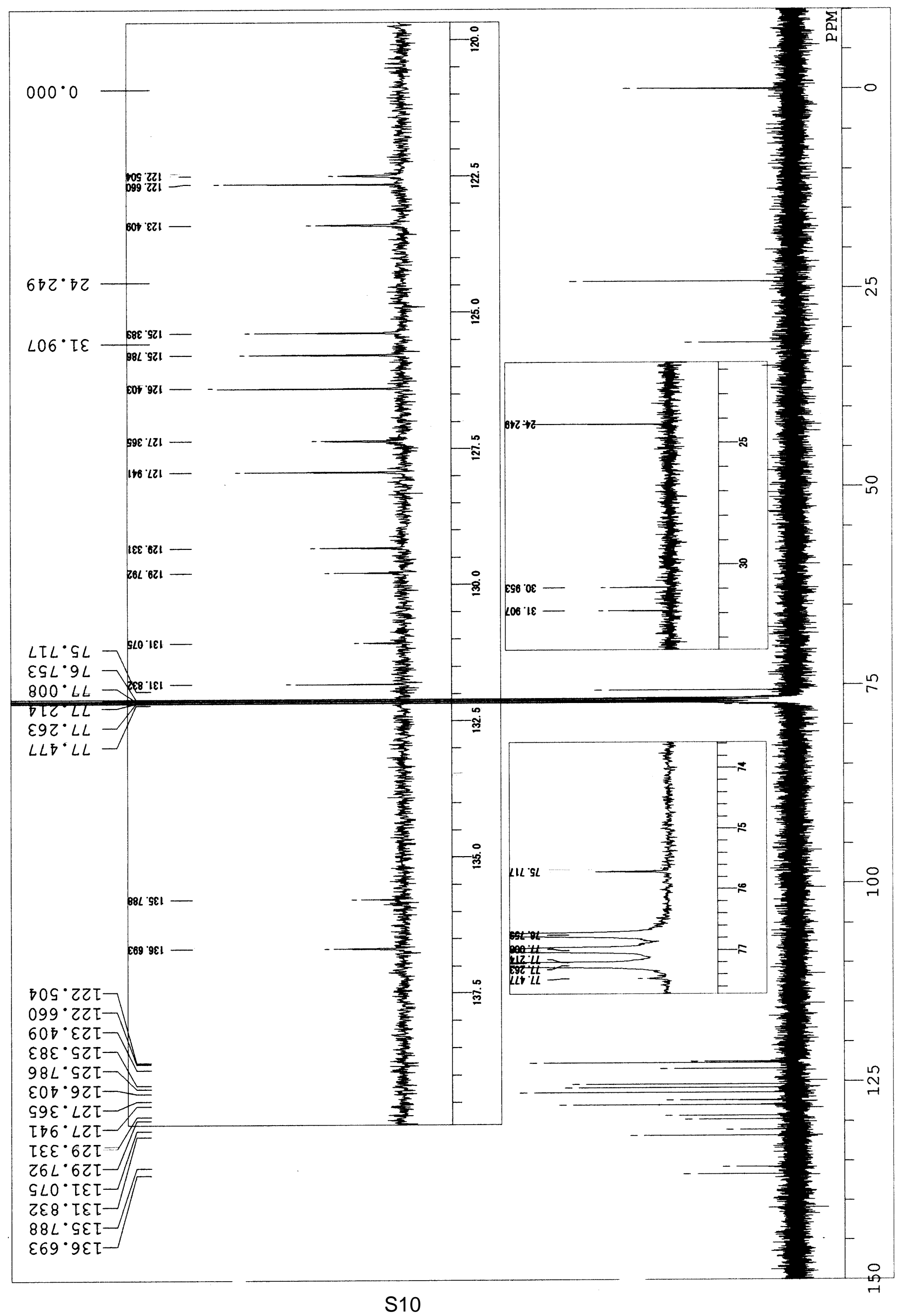


${ }^{1} \mathrm{H}$ NMR of syn-7 in $\mathrm{CDCl}_{3}$

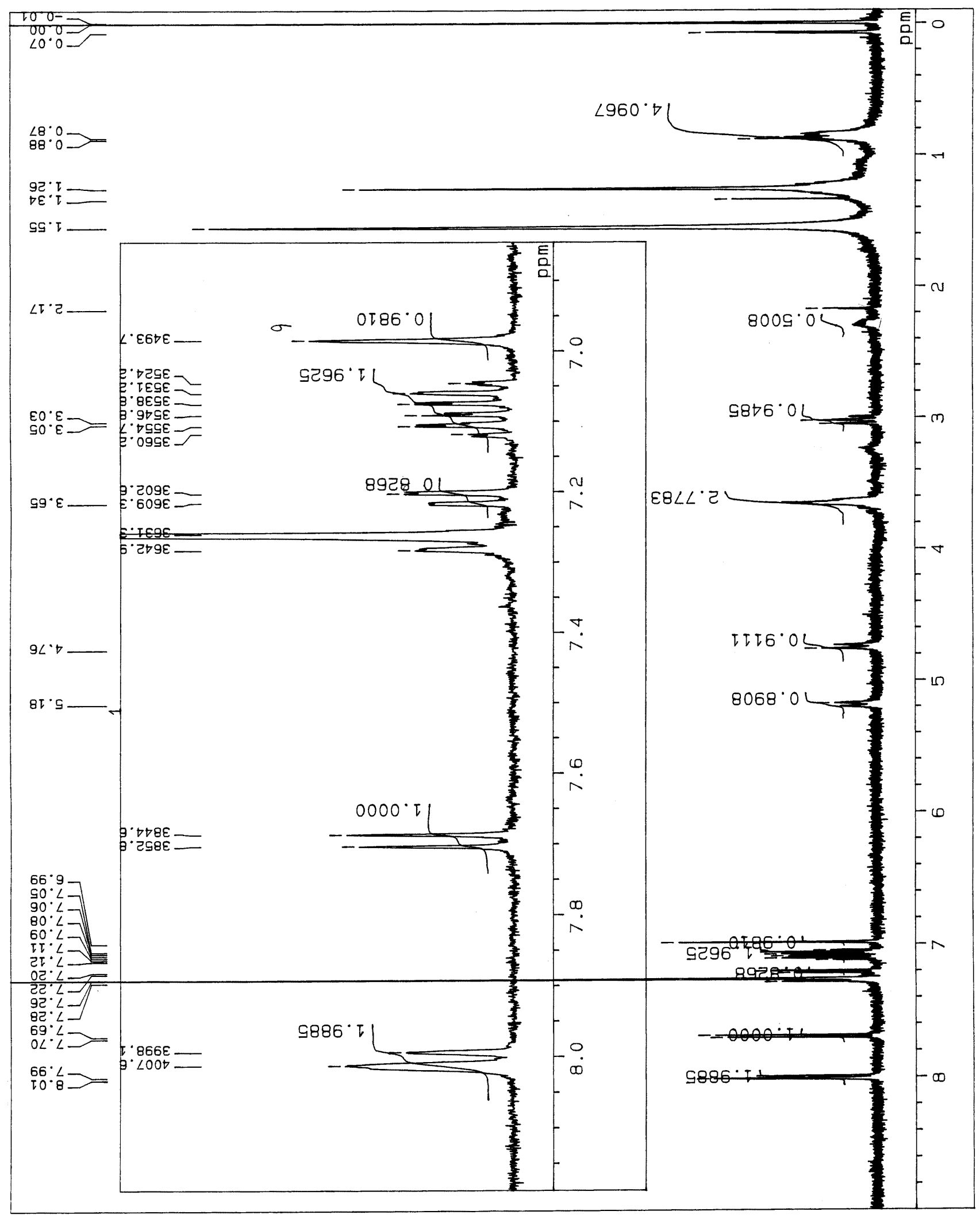


${ }^{13} \mathrm{C}$ NMR of syn-7 in $\mathrm{CDCl}_{3}$

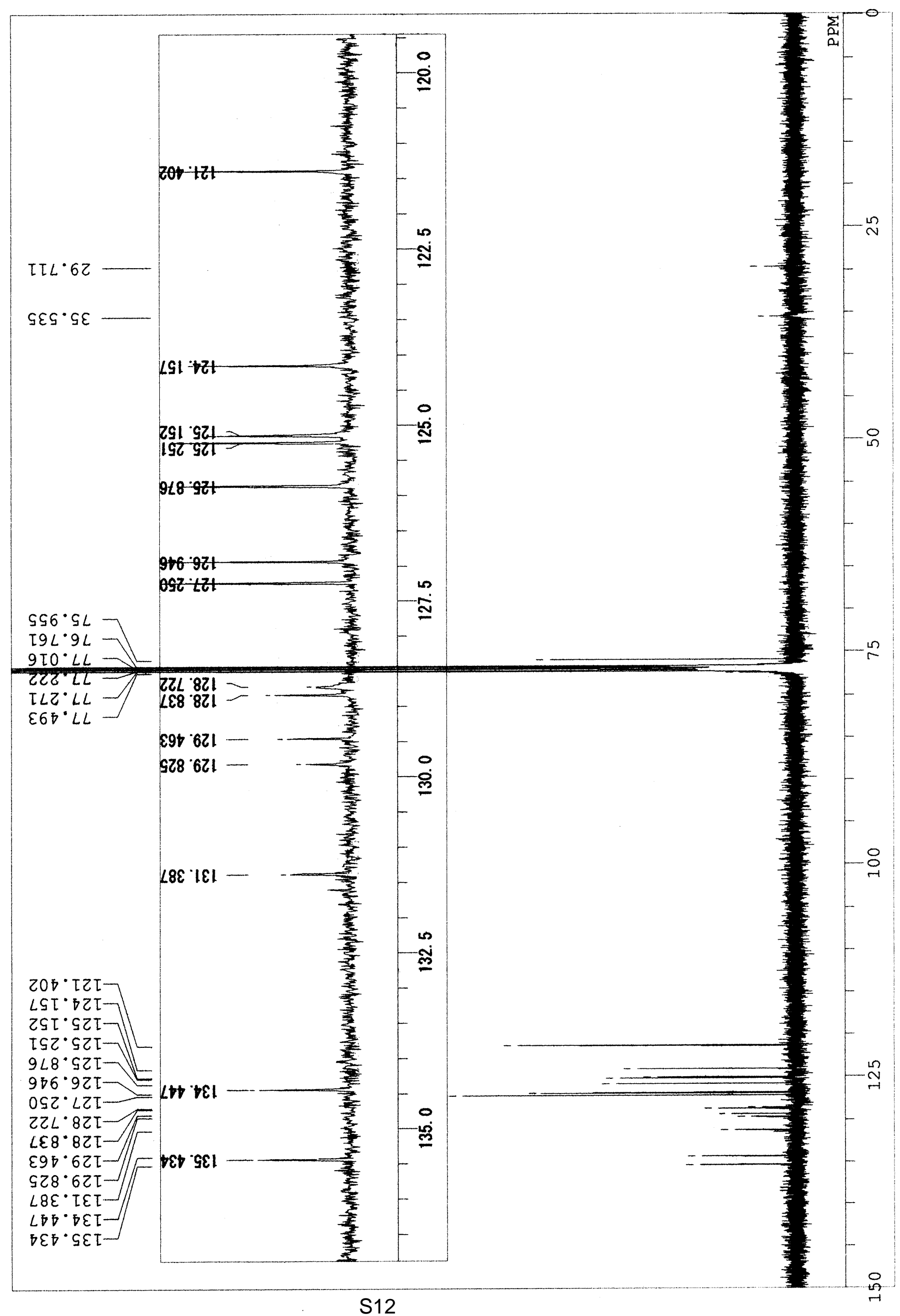

